\title{
La compleja relación entre tradiciones discursivas y estilo
}

\author{
Alfonso Gallegos SHIBYA \\ Universidad de Guadalajara (UDG)
}

\section{ఠ \\ OPEN ACCESS \\ EDITADO POR \\ - Valéria Severina Gomes (UFRPE) \\ - Aurea Zavam (UFC) \\ - Konstanze Jungbluth (EUV)}

REVISADO POR

- Eliabe Procópio (UFRR)

- Mizael do Nascimento (UFRPE)

FECHAS

- Recibido: 22/08/2020

- Acepto: 08/11/2020

- Publicado: 17/12/2020

COMO CITAR

Shibya, A. G. (2020)

La compleja relación entre

tradiciones discursivas y estilo.

Revista da Abralin, v. 19, n. 3, p. 568-581, 2020.

\section{RESUMEN}

El modelo de tradiciones discursivas, surgido en la romanística alemana, ha ofrecido una nueva perspectiva de análisis para gran cantidad de estudios lingüísticos (tanto diacrónicos como sincrónicos); sin embargo, no existe un acuerdo definitivo sobre su extensión y límites. En este trabajo se analiza la relación entre tradiciones discursivas y estilo, para lo que se propone (a) una clasificación de las tradiciones discursivas en dos tipos, con base en la incidencia (o no) de factores tipológicos en su tranmisión interlingüística, y (b) una distinción entre estilo individual y supraindividual. A partir del análisis de varios casos concretos, se argumenta que solo el estilo supraindividual corresponde a un tipo de tradición discursiva. Los estilos suprainviduales se basan en configuraciones funcionales genéricas interlingüísticas, que cada lengua actualiza de acuerdo con sus características tipológicas. De esta manera, los conceptos de tradición discursiva y estilo no son equivalentes, sino que se intersectan parcialmente.

\section{RESUMO}

O modelo de tradições discursivas, surgido na romanística alemã, tem oferecido uma nova perspectiva de análise para grande quantidade de estudos linguísticos (tanto diacrônicos como sincrônicos); sem dúvida, não existe um acordo definitivo sobre sua extensão e limites. Neste trabalho se analisa a relação entre tradições discursivas e estilo, para o que se propõe (a) uma classificação das tradições discursivas em dois tipos, com base na incidência (ou não) de fatores tipológicos em sua transmissão interlinguística, e (b) uma distinção entre estilo individual e supraindividual. A partir da análise de vários casos concretos, argumenta-se que só o estilo supraindividual corresponde a um tipo de tradição discursiva. Os estilos 


\section{REVISTA DA ABRALIN}

suprainviduais se baseiam em configurações funcionais genéricas interlinguísticas, que cada língua atualiza de acordo com suas características tipológicas. Desta maneira, os conceitos de tradição discursiva e estilo não são equivalentes, mas que se interseccionam parcialmente.

\section{ABSTRACT}

The discourse traditions model, originated in the German Romance linguistics, has offered a new analytical perspective for many linguistic studies (both diachronic and synchronic). However, there is not a definite consensus about its extensions and limit. This paper examines the relationship between discourse traditions and style. To this aim, I propose (a) a classification of discourse traditions into two types, based on the influence (or lack thereof) of typological factors in their interlinguistic transmission, and (b) a distinction between individual and supraindividual style. After the analysis of various concrete cases, I argue that the supraindividual style corresponds to a type of discourse tradition. Supraindividual styles are based on interlinguistic generic functional configurations, which each language realizes according to its typological characteristics. Thus, the notions of discourse tradition and style, albeit not equivalent, partially intersect each other.

\section{PALABRAS CLAVE}

Tradiciones discursivas. Estilo. Lengua individual. Aspectos idiomáticos y supraidiomáticos.

\section{PALAVRAS-CHAVE}

Tradições discursivas. Estilo. Língua individual. Aspectos idiomáticos e supraidiomáticos.

\section{KEYWORDS}

Discourse traditions. Style. Individual language. Idiomatic and supraidiomatic aspects. 


\section{REVISTA DA ABRALIN}

\section{Introducción}

Durante las últimas décadas, los estudios de las lenguas romances se han visto enriquecidos con el modelo de las tradiciones discursivas ${ }^{1}$. Esta propuesta se basa en el postulado de que "hablar no es sólo decir algo a alguien sobre las cosas de acuerdo con las reglas de un lengua (esto es, su sistema y su norma), sino que suele ser, además, decir algo según una determinada tradición textual de hacerlo" (KABATEK, 2005, p. 156). Las tradiciones discursivas representan modelos o esquemas histórico-contingentes que guían la producción y recepción de cualquier texto o discurso y que, si bien rebasan el marco de las lenguas individuales, seleccionan en estas, al menos parcialmente, el uso de una variedad lingüística o modelos de habla determinados (cf. OESTERREICHER 1997).

El modelo de las tradiciones discursivas supone que las lenguas históricas pueden ampliar paulatinamente sus espacios funcionales hacia todo tipo de situaciones comunicativas, lo que presupone (1) que son capaces de generar, adaptar o incorporar cualquier tradición discursiva (independientemente de su nivel de complejidad, cf. WILHELM, 2001), y (2) que pueden desarrollar los recursos lingüísticos necesarios para la consecución de tales fines expresivos (cf. BOSSONG, 1979; RAIBLE, 1985; KABATEK, 2005; KOCH, 2008). Esta propuesta ha permitido abordar una serie de fenómenos lingüísticos desde una perspectiva distinta, como la relación entre géneros textuales, variedades de lengua y cambio lingüístico (cf. KABATEK, 2005); el desarrollo de la escrituralidad de las lenguas (cf. FRANK-JOB, 2003; WILHELM, 2003); las tradiciones discursivas y la traducción (cf. ALBRECHT, 2003), etc.

Si bien el modelo de las tradiciones discursivas ha sido de una enorme utilidad en una multiplicidad de estudios lingüísticos, tanto sincrónicos como diacrónicos, no existe un acuerdo definitivo sobre los elementos que debe abarcar este concepto. Uno de los aspectos controversiales dentro del modelo es la relación entre estilo y tradiciones discursivas, sobre lo que es posible distinguir dos posturas bien diferenciadas:

a) Algunos investigadores sostienen que los estilos no son tradiciones discursivas, sino variedades diafásicas (o registros) que se ubican en el marco de las lenguas históricas individuales, por lo que forman parte del saber idiomático de los hablantes (cf. COSERIU, 1982; LÓPEZ SERENA, 2012; KABATEK, 2018a).

b) Otros investigadores, en cambio, señalan que los estilos son tradiciones discursivas al igual que los tipos textuales, los géneros, las formas de conversación, los actos de habla, etc., y por ello comparten el mismo carácter supraidiomático (cf. KOCH, 1997; KABATEK, 2007; KABATEK, 2018b).

\footnotetext{
${ }^{1}$ Este modelo se remonta a un trabajo de Schlieben-Lange (1983) sobre pragmática histórica, aunque la sistematización y el deslinde teórico fueron llevados a cabo por Koch (1997) y Oesterreicher (1997).
} 


\section{REVISTA DA ABRALIN}

Esta falta de consenso se debe, entre otras cosas, a los diferentes conceptos de 'estilo' que asumen cada uno de los autores. Por tal motivo, resulta metodológicamente indispensable deslindar este término para establecer después las interrelaciones existentes entre el estilo y las tradiciones discursivas.

Con el término 'estilo' me refiero al conjunto de rasgos específicos de un acto discursivo que resultan de las elecciones de los hablantes con respecto a las posibilidades que ofrece una lengua determinada tanto en el léxico como en la gramática: vocabulario, colocaciones, construcciones sintácticas, morfología derivativa, combinación de frases, etc. (cf. BROWN; MILLER, 2013). Definido 'estilo' de esta manera, podemos distinguir entonces dos niveles diferentes: (i) individual y (ii) supraindividual (véase infra, §3). La tesis de este trabajo es que el estilo, solo en el sentido supraindividual, corresponde a un tipo de tradición discursiva. De esta manera, los conceptos de 'estilo' y ‘tradición discursiva', sin ser equivalentes, quedan parcialmente traslapados.

En las siguientes secciones abordaré algunos aspectos teóricos relevantes del modelo de las tradiciones discursivas $(\S 1)$, para después proponer una clasificación de las tradiciones discursivas de acuerdo con los aspectos tipológicos que condicionan su dinámica de transmisión interlingüística (§2). Posteriomente, analizaré las interrelaciones entre tradiciones discursivas y estilo (§3), y finalmente expondré algunas conclusiones $(\S 4)$.

\section{El modelo de las tradiciones discursivas}

Los trabajos sobre tradiciones discursivas remiten generalmente a la distinción propuesta por Coseriu (1981) de tres niveles de lo lingüístico, cada uno de los cuales corresponde a un diferente grado de generalización y abstracción: (i) el 'nivel universal' está relacionado con la actividad del hablar en general como hecho filogenético o antropológico sin determinación histórica alguna; (ii) el 'nivel histórico' corresponde a las lenguas particulares (como el portugués, el japonés, el pirahã, etc., y sus respectivas variedades), y (iii) el 'nivel individual' se entiende como los actos lingüísticos concretos realizados por personas en situaciones determinadas. A cada uno de estos niveles es posible atribuir un tipo de actividad, de saber ('técnica') y de producto:

\begin{tabular}{l|l|l|l}
\hline \multicolumn{1}{c|}{ NIVEL } & \multicolumn{2}{c}{ ASPECTO } & \multicolumn{1}{c}{ SABER } \\
\hline universal & ACTIVIDAD & \multicolumn{1}{c}{ PRODCTO } \\
\hline histórico & hablar en general & saber elocucional & totalidad de los enunciados \\
\hline individual & producir un discurso & saber idiomático & lengua \\
\hline
\end{tabular}




\section{REVISTA DA ABRALIN}

Algunos autores, sin embargo, han señalado la necesidad de una diferenciación en el nivel histórico, ya que el saber expresivo comprende no sólo el dominio de la gramática y léxico de una lengua determinada, sino también la capacidad de producir textos según modelos históricos que, en principio, son independientes del saber idiomático de las lenguas individuales ${ }^{2}$. Considérese, por ejemplo, que tradiciones textuales como la carta, la novela, el artículo científico, la crónica, etc. no están asociadas a ninguna lengua en particular -y su identidad incluso es reconocible más allá de los límites de las lenguas individuales. Por tal motivo, Koch (1997) y Oesterreicher (1997) proponen desdoblar el nivel histórico coseriano para distinguir claramente (i) la historicidad de la lengua (es decir, la gramática y el léxico de una lengua con su respectiva norma) de (ii) la historicidad de los textos (es decir, las tradiciones discursivas), aunque "la historicidad primaria de la lengua y la historicidad secundaria de los textos, la tradicionalidad, están relacionadas íntimamente" (KABATEK, 2007, p. 345) ${ }^{3}$.

\begin{tabular}{l|l|l}
\hline \multicolumn{1}{c|}{ NIVEL } & \multicolumn{1}{c|}{ DOMINIO } & \multicolumn{1}{c}{ TIPO DE REGLAS } \\
\hline universal & actividad del hablar & reglas elocucionales \\
\hline \multirow{2}{*}{ histórico } & lengua histórica particular & reglas idiomáticas \\
\cline { 2 - 3 } & tradición discursiva & reglas discursivas \\
\hline actual/individual & discurso & \\
\hline \multicolumn{2}{c}{ ESQUEMA 2 - Niveles de lo lingüístico (modelo ampliado) } \\
\multicolumn{2}{c}{ Fuente: Koch (2008, p. 54) }
\end{tabular}

Las tradiciones discursivas consisten pues en "modelos normativos convencionalizados de la transmisión lingüística de sentido, que regulan la producción y la recepción de discursos"4 (OESTERREICHER, 1997, p. 20), por lo que la elaboración de un texto implica necesariamente considerar tanto las reglas de una lengua histórica (es decir, una gramática y un léxico particulares) como una determinada tradición discursiva. Estos modelos convencionalizados pueden desarrollarse a partir de cualquier elemento tanto de expresión como de contenido, y es precisamente esta repetición la que establece un vínculo semiótico entre un acto de enunciación concreto y una determinada tradición discursiva (cf. KABATEK, 2005).

Las tradiciones discursivas, en principio, no pertenecen a una lengua en particular. La relación existente entre una tradición discursiva y una determinada comunidad lingüística es el resultado de

\footnotetext{
${ }^{2}$ Véase en este sentido Schlieben-Lange (1983); Coseriu (1992 [1988]); Koch (1997); Oesterreicher (1997).

${ }^{3}$ Una diferencia más en la historicidad de ambas entidades radica en que, mientras los grupos constitutivos de las lenguas históricas son comunidades lingüísticas, los de las tradiciones discursivas están definidos por variables socioculturales como profesiones, religiones, corrientes literarias, grupos políticos, actividades artísticas y lúdicas, etc. (cf. SCHLIEBEN-LANGE, 1983 ; COSERIU, 1992 [1988]; KOCH, 1997). La orientación de la delimitación también es distinta, ya que "las lenguas históricas (o sus variedades) definen a los grupos (es decir, las comunidades lingüísticas), mientras que son los grupos (profesionales, religiosos, literarios, etc.) los que definen a las tradiciones discursivas" (KOCH, 2008, p. 55).

4 Original en alemán: "normative, die Diskursproduktion und Diskursrezeption steurende, konvensionalisierte Muster der sprachlichen Sinnvermittlung" (traducción propia).
} 


\section{REVISTA DA ABRALIN}

eventos contingentes, ya que la historicidad de las reglas idiomáticas y de las discursivas es de naturaleza distinta:

\footnotetext{
Los aspectos históricos del saber expresivo pueden superar en extensión las comunidades idiomáticas abarcando varias de ellas (ser, por ejemplo, propios de la 'cultura occidental'), superar los límites de las comunidades idiomáticas sin abarcarlas (ser, por ejemplo, propios de los estratos cultos de las comunidades 'occidentales'), corresponder a comunidades menores dentro de las comunidades idiomáticas; y pueden hasta coincidir con las comunidades idiomáticas, en la medida en que los límites de ciertos hechos de experiencia o de cultura coincidan, precisamente, con los límites de esas comunidades. (COSERIU, 1956-1957, apud LÓPEZ SERENA, 2012, p. 270-271)
}

Ahora bien, el hecho de que las tradiciones discursivas sean supraidiomáticas tampoco significa que cualquiera de ellas puede 'emigrar' sin más de una lengua a otra; por lo contrario, en muchos casos la lengua huésped necesita realizar complejos procesos de acomodación o asimilación que le permitan incorporar algún modelo discursivo. En vista de ello, quisiera proponer dos tipos diferentes de tradiciones discursivas, considerando la dinámica que subyace a su transmisión de una lengua a otra. Esta clasificación nos servirá posteriormente para ubicar el estilo en este modelo teórico.

\section{Dos tipos de tradiciones discursivas}

Un grupo de tradiciones discursivas, a las que llamaré tradiciones discursivas de TIPO I, lo constituyen todas aquellas que pueden pasar libremente de una lengua a otra porque se basan en elementos de forma o de contenido que no dependen de las características tipológicas de ninguna lengua en particular. De esta manera, el soneto, el artículo científico, la fábula, la editorial, etc. no se limitan a lenguas como el italiano, griego, español, inglés, etc., sino que podrían realizarse, en principio, en cualquiera otra (independientemente de su configuración tipológica). Lo mismo puede afirmarse de las tradiciones discursivas basadas en la repetición de ciertos aspectos de contenido; por ejemplo, las obras basadas en topoi tales como el don Juan, el amor caballeresco, el descenso a los infiernos, el locus amoenus y el locus horridus, el viaje de regreso a la patria, etc.

A diferencia de estas, existen otras tradiciones discursivas, que denominaré de TIPO II, que sí dependen de rasgos tipológicos, y precisamente por eso no pueden transferirse libremente a cualquier lengua. Un caso serían los modelos preceptivos de la poesía clásica griega o latina, basados en unidades métricas llamadas 'pies', formados por un número reducido de sílabas breves y largas (de dos a cuatro). Las unidades de medida para cada sílaba se denominan 'moras' - una para las sílabas cortas, dos para las largas-, y su combinación crea estructuras métricas fijas de dos sílabas (pirriquio o díbraco, yambo, troqueo y espondeo), tres sílabas (tríbraco, dáctilo, anfíbraco, anapesto, etc.) y cuatro sílabas (tetrabraquio o proceleusmático, ditroqueo, diyambo, coriambo, antispasto, etc.) (cf. BROGAN; COLE, 1993). Estos modelos textuales no pueden ser incorporados sin más por lenguas que no distinguen la diferencia fonológica entre vocales cortas y largas. En este sentido, lo que hicieron 


\section{REVISTA DA ABRALIN}

históricamente lenguas como el español o el portugués fue sustituir el pie latino con la sílaba como unidad métrica, utilizando un ritmo combinatorio de tónicas-átonas por analogía con el ritmo cuantitativo de largas-cortas (cf. BROGAN, 1993).

Un ejemplo aún más espectacular de tradiciones discursivas asociadas a rasgos tipológicos está representado por la poesía tailandesa, que recurre a una sofisticadísima métrica rítmica de tonos para generar efectos poéticos ${ }^{5}$. El tailandés es una lengua que distingue cinco tonos fonológicos bajo, medio, alto, descendente y ascendente-, y su poesía adopta ciertas reglas para rima interior (es decir, en el mismo verso) y rima exterior (entre versos de la misma estrofa o de estrofas diferentes). Uno de los poemas mas básicos y populares desde la época antigua es el octosílabo, que se puede representar de la siguiente manera:

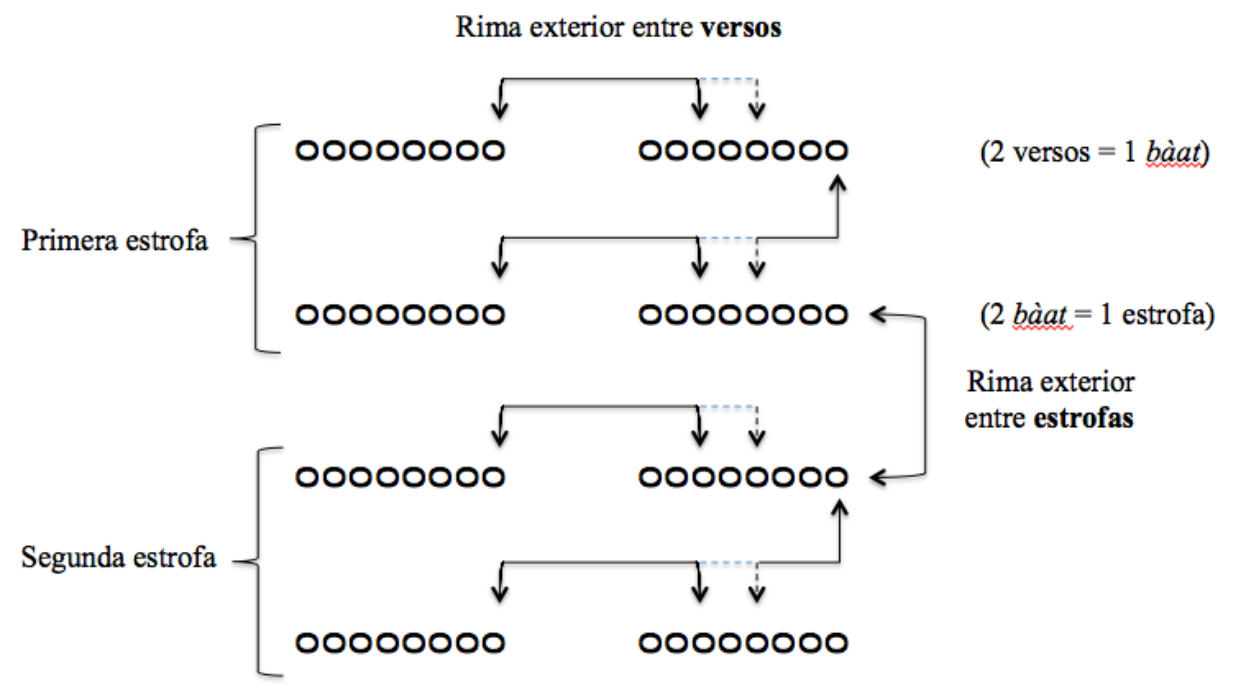

ESQUEMA 3 - Estructura del poema octosílabo tailandés Fuente: Tumtavitikul (2001, p. 30) y Chaichumporn (2013, p. 3)

En este género textual, ocho sílabas forman un verso, y dos versos un bàat (o baht); la estrofa, a su vez, está formada por dos bàat. El poema octosílabo en cuestión está integrado por dos estrofas (para un total de cuatro bàat, o bien ocho versos). Los tonos están determinados de tal manera que en el primer verso puede usarse cualquiera de los cinco tonos; en el segundo verso no se utilizan los tonos medio ni alto, mientras que en los versos tres y cuatro se usan solamente tonos medio y alto. Para complicar aún más las cosas, hay especificaciones muy estrictas en cuanto a la rima interior (en el mismo verso) y exterior (entre diferentes versos). En cuanto a la interior, la quinta palabra rima generalmente con la séptima palabra de cada verso. Y con respecto a la rima exterior, se debe seguir el siguiente modelo:

\footnotetext{
${ }^{5}$ Agradezco a Jaruchai Chaichumporn que me haya señalado estas particularidades de la poesía tailandesa (comunicación personal).
} 


\section{REVISTA DA ABRALIN}

- la última palabra del primer verso rima con la tercera o la quinta del segundo verso;

- la última palabra del segundo verso rima con la última palabra del tercer verso, así como con la tercera o quinta palabra del cuarto verso;

- la última palabra del cuarto verso rima con el segundo verso de la siguiente estrofa, de manera que la métrica rítmica de ambas estrofas queda enlazada;

- la métrica rítmica no permite vincular vocales cortas con largas, sino que vocales cortas deben rimar con otras del mismo tipo (cf. HUDAK, 1986; TUMTAVITIKUL, 2001; CHAICHUMPORN, 2013).

Como podemos apreciar, la poesía tailandesa contiene una compleja relación entre la métrica rítmica y el tono. Ahora bien, si una lengua no dispone de las mismas posibilidades fonológicas que el tailandés, ¿cómo podría adoptar esta tradición discursiva? En principio, otras lenguas tonales serían las mejores candidatas para hacerlo, pero esto no significa que carecerían de dificultades, dado que su sistema tonal no sería necesariamente paralelo al del tailandés ${ }^{6}$. ¿Y qué decir sobre lenguas no tonales como el inglés, el turco, el coreano, el náhuatl, etc.? Por supuesto que podrían incorporar este modelo textual -tal como las lenguas romances asimilaron, con modificaciones, la preceptiva poética latina-, pero requerirían muchísima adaptación (y creatividad) para llevar a cabo semejante empresa.

En resumen, las tradiciones discursivas tienen una historicidad diferente a la de las lenguas individuales. Aunque son supraidiomáticas, en el sentido de que no están limitadas al espacio de cada lengua, esto no implica que puedan trasladarse libremente de una a otra: las tradiciones discursivas que he llamado de TIPO II están asociadas con rasgos tipológicos que condicionan su asimilación.

\section{El estilo y las tradiciones discursivas}

He señalado previamente que al hacer referencia a 'estilo' me refiero al conjunto de rasgos específicos de un discurso que resultan de las elecciones de los hablantes con respecto a las posibilidades (tanto gramaticales como léxicas) de una lengua. Ahora bien, al igual que las tradiciones discursivas, es necesario distinguir dos tipos de estilo que remiten a diferentes niveles de lengua. El estilo puede

\footnotetext{
${ }^{6}$ Las lenguas con sistemas tonales simples disponen esencialmente de solo un contraste binario entre tonos alto y bajo (como el tibetano, ewe, rutul, khmu', etc.). Otras lenguas cuentan en sus sistemas fonológicos con tres, cuatro o más tonos diferentes (cf. MADDIESON, 2005).
} 


\section{REVISTA DA ABRALIN}

ser individual, es decir, una configuración que corresponde al conjunto de reglas idiosincráticas del hablar (cf. KOCH, 2008). Si se considera, por ejemplo, el estilo individual de un autor, las elecciones permiten reconocer un uso particular de la lengua, y con ello textos de Jorge Luis Borges, José Saramago, William Faulkner, etc. La posibilidad de distinguir el uso individual de la lengua a partir de la selección de rasgos específicos es también el postulado fundamental de la lingüística forense.

No obstante, existen también estilos supraindividuales que preestablecen ciertas pautas para la realización de determinados tipos de discurso: hablamos de estilo científico, técnico, periodístico, religioso, jurídico, etc. pero también de barroco, manierismo, modernismo, etc. Estas variantes representan tradiciones de habla históricas que regulan la producción y la recepción del discurso, y están vinculadas con ciertos grupos establecidos a partir de una multiplicidad de variables. La pregunta sería, pues, si los estilos, concebidos de esta manera, deben ser entendidos (o no) como tradiciones discursivas. Pues bien, el estilo individual no representa per se una tradición discursiva, ya que se trata de un ámbito expresivo idiosincrático que no tiene ninguna historicidad idiomática ni discursiva. En cambio, el estilo supraindividual -esto es, la elección de determinadas pautas de uso de la lengua asociadas a ciertos ámbitos sociales (periodistas, científicos, técnicos, etc.) o corrientes literarias (romanticismo, modernismo, etc.)- representa una tradición discursiva de TIPO II, y está por ello sometido a las mismas dinámicas que las demás tradiciones discursivas de su clase.

Quisiera ejemplificar lo anterior a partir del estilo de los textos especializados (por ejemplo, científicos y técnicos). El estilo de los lenguajes especializados no se desarrolla ex nihilo al interior de los marcos idiomáticos de las lenguas históricas, sino que responde a ciertos principios funcionales que sobrepasan las fronteras de las lenguas individuales (BENEŠ, 1969). A este carácter supraidiomático se debe una serie de parámetros tales como la tendencia a la precisión y concisión, el predominio de la función referencial, la planificación macroestructural, la relación entre texto e imagen, la interacción con otros sistemas semióticos, la despersonalización del discurso, la reflexividad (esto es, la metainformación) del texto: definiciones, explicaciones, precisiones, introducción de términos, indicadores de estructuración, etc. Se trata, pues, de una configuración genérica que conduce a las siguientes tendencias verbalizadoras en las lenguas europeas (cf. KALVERKÄMPER, 1998; SCHMITT, 1992; SCHIFKO, 2001):

- Creación de términos especializados mediante terminologización, formación de palabras (derivación, composición, conversión), abreviaciones, siglas y préstamos de distintos tipos (calcos formales y de significado), entre otras.

- Establecimiento de referencia a partir de sintagmas nominales con fuerte carga de determinación (tanto extensional como intensional).

- Reificación de contenidos proposicionales mediante el uso de formas verbales no-finitas (gerundios, participios, infinitivos) (cf. LEHMANN, 1982).

- Uso frecuente de la técnica de nominalización (cf. GALLEGOS SHIBYA, 2010). 


\section{REVISTA DA ABRALIN}

- Formas de pasiva como recurso de desagentivización de la estructura semántica de las proposiciones.

- Técnicas morfosintácticas de despersonalización (formas reflexivas, apersonales, impersonales, etc.).

- Uso de verbos que cumplen funciones sintácticas pero que muestran cierto vaciamiento semántico (como en español efectuar, proceder, conferir, basar(se), hacer, ofrecer, permitir, introducir, establecer, realizar(se), conferir, etc.), sobre todo al ser usados en estructuras verbales funcionales.

Si bien algunos de estos modelos verbalizadores pueden ser encontrados, en mayor o menor medida, en textos técnicos y científicos de muy diversas lenguas -como el uso de la voz pasiva para despersonalizar el texto o la condensación de información por medio de la nominalización-, la selección de rasgos idiomáticos para este estilo dependerá finalmente de las características tipológicas de la lengua en cuestión ${ }^{7}$. Ahora bien, a pesar de estas diferencias en la selección de rasgos, los lenguajes especializados de las diversas lenguas pueden considerarse como pertenecientes a un mismo estilo, ya que responden todos ellos a los mismos parámetros funcionales.

El siguiente caso ejemplificará mejor la dinámica interlingüística del estilo como tradición discursiva de TIPO II. Consideremos uno de los lenguajes especializados del español, el jurídico, y su traducción al huichol ${ }^{8}$. Los textos jurídicos en español (y otras lenguas europeas) tienen un estilo caracterizado, entre otros atributos, por una configuración discursiva argumentativa, exhaustividad referencial, abundante uso de terminología (juicio, demanda, amparo, fiscalía, media filiación, etc.), empleo particular de estilos directo e indirecto, intertextualidad, etc. (cf. ALCARAZ VARO; HUGHES, 2009). Se ha señalado, sin embargo, que las tendencias de los textos jurídicos responden en gran medida a las características tipológicas de las lenguas europeas, que se caracterizan más por un estilo nominal, mientras que "el huichol y otras lenguas americanas son lenguas centradas más en el evento que en los participantes o los objetos" (ITURRIOZ; RAMÍREZ, 2012, p. 143). El huichol es una lengua con un carácter muy centralizante, esto es, que tiende a "acumular la mayor parte de la información en el predicado, generalmente verbal, a costa de las restantes clases de palabras" (ITURRIOZ; GÓMEZ; RAMÍREZ, 2004a, p. 153), de manera tal que la palabra predicativa tiende a ser coextensiva con el enunciado. Estas características tipológicas tienen consecuencias muy importantes,

\footnotetext{
${ }^{7}$ En este sentido, se ha señalado que los modelos verbalizadores anteriormente señalados son consecuencia de las características tipológicas de las lenguas indoeuropeas, por lo que su valor universal ha sido puesto en duda (cf. SCHIFKO, 2001)

${ }^{8}$ Lengua de la familia yuto-azteca hablada por 49,509 personas en el occidente de México (de acuerdo con el XII Censo de Población y Vivienda 2010, http://atlas.inpi.gob.mx/?page id=815; fecha de consulta: 20/10/2020).
} 


\section{REVISTA DA ABRALIN}

como la reducción del número de constituyentes sintácticos nominales por elisión o incorporación, ya que resultan prescindibles en virtud de la información codificada en el predicado; la disminución del número de constituyentes sintácticos por clitización y morfologización; la reducción de la complejidad sintáctica y morfológica de los constituyentes no predicativos, etc. (cf. ITURRIOZ; GÓMEZ; RAMÍREZ, 2004b).

El reto se encuentra, pues, en traducir un texto de estilo jurídico a una lengua (a) que no dispone aún de esta tradición discursiva, y (b) que presenta características tipológicas muy diferentes a las lenguas europeas. Por tal motivo,

\footnotetext{
donde el español requiere un término técnico nominal, el huichol puede prescindir de él, porque puede expresar lo mismo dentro de la palabra predicativa [...] ¿Cómo se traduce del español, una lengua predominantemente nominal, fusionante y menos explícita, a una lengua como huichol polisintética, centralizante, verbalizante y explicitante, que tiende a hacer coextensivos la oración y el predicado? (ITURRIOZ; RAMÍREZ, 2012, p. 138 y 142)
}

Precisamente por sus rasgos tipológicos, el huichol tiene que seleccionar determinadas técnicas lingüísticas, que no coinciden necesariamente con las del español u otras lenguas europeas, para cristalizar la configuración genérica del estilo jurídico (esto es, precisión referencial de participantes y eventos, despersonalización, argumentación, uso de discurso directo e indirecto, etc.). De esta manera, el carácter supraidiomático de los estilos no radica en la selección de determinados modelos verbalizadores o técnicas lingüísticas -que pueden variar en virtud de los rasgos tipológicos de cada lengua-, sino en la transmisión e incorporación de configuraciones discursivas que tienen un valor funcional y que, en última instancia, orientan a los hablantes en la selección de rasgos idiomáticos para la elaboración de textos vinculados a ciertos ámbitos socioculturales y corrientes literarias.

\section{Reflexiones finales}

¿Es posible, entonces, entender el estilo como un tipo de tradición discursiva? La respuesta es que no existe una identificación plena entre ambos conceptos. Por una parte, el estilo excede el ámbito de las tradiciones discursivas en tanto que abarca también el 'estilo individual' (resultado de elecciones idiosincráticas y elementos expresivos que no están incluidas en aquellas). Por otra, el 'estilo supraindividual' responde a la dinámica de las tradiciones discursivas de TIPO II, es decir, de modelos funcionales interlingüísticos a los que cada lengua responde de acuerdo con sus características tipológicas. La relación entre tradiciones discursivas y estilo aquí propuesta se puede apreciar mejor en el siguiente esquema: 


\section{REVISTA DA ABRALIN}

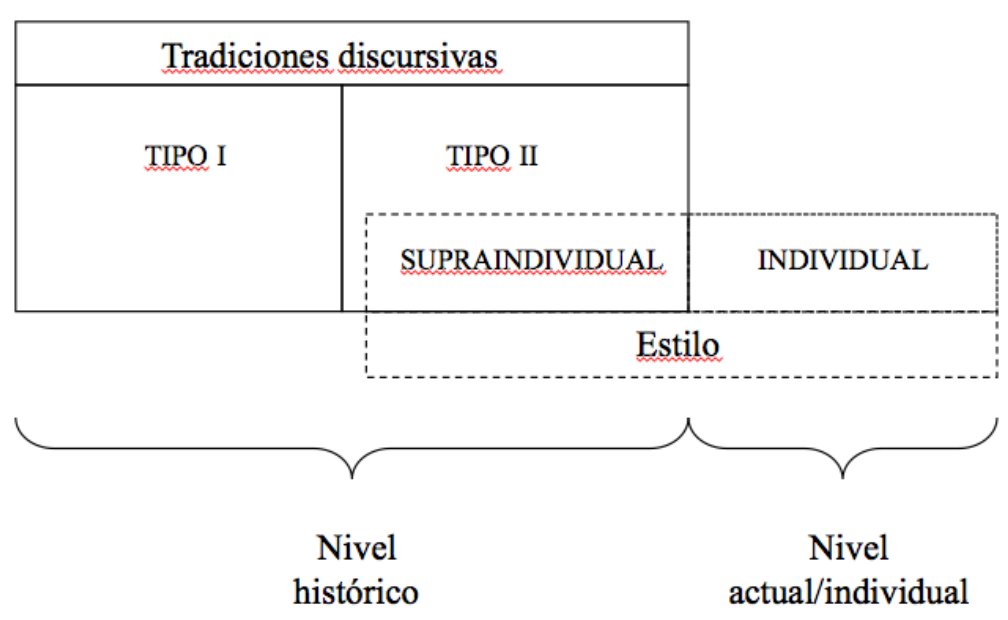

ESQUEMA 4 - Relación entre tradiciones discursivas y estilo

Fuente: Elaborado por el autor

Cuando una lengua, debido a necesidades comunicativas específicas, tiene que adoptar cierto estilo funcional (técnico, científico; barroco, modernismo, etc.), puede recurrir a alguna de las siguientes dos estrategias: (a) una tendencia asimilativa, es decir, intentar incorporar en su acervo las estrategias lingüísticas de la lengua fuente, $\mathrm{o}(\mathrm{b})$ una tendencia acomodativa, en la cual se favorecen las técnicas lingüísticas de las que dispone la propia lengua meta para dar respuesta a esos fines comunicativos (cf. ITURRIOZ; RAMÍREZ, 2012). En cualquier caso, el desarrollo de estilos funcionales para los diferentes ámbitos discursivos implica un proceso histórico por el cual la lengua en cuestión amplía paulatinamente su presencia hacia esferas más alejadas de la vida cotidiana como el derecho, los medios de comunicación, la educación, la ciencia y la técnica, etc.

\section{REFERENCIAS}

ALBRECHT, Jörn. Können Diskurstraditionen auf dem Wege der Übersetzung Sprachwandel auslösen? In: Aschenberg, Heidi; Wilhelm, Raymund (eds.). Romanische Sprachgeschichte und Diskurstraditionen. Tübingen: Gunter Narr, p. 37-53, 2003.

ALCARAZ VARÓ, Enrique; HUGHES, Brian. El español jurídico. Barcelona: Ariel, 2009.

BENEŠ, Eduard. Zur Typologie der Stilgattungen der wissenschaftlichen Prosa. Deutsch als Fremdsprache, Berlin, v. 6, n. 3, p. 225-233, 1969.

BOSSONG, Georg. Probleme der Übersetzung wissenschaftlicher Werke aus dem Arabischen in das Altspanische zur Zeit Alfons des Weisen. Tübingen: Niemeyer, 1979. DOI: https://doi.org/10.1515/9783111590189

BROGAN, Terry V. F. Prosody. In: Preminger, Alex; Brogan, Terry V. F. (eds.). The New Princenton Encyclopedia of Poetry and Poetics. Princenton: Princenton University Press, p. 982-994, 1993. 


\section{REVISTA DA ABRALIN}

BROGAN, Terry V. F.; COLE, A. Thomas. Classical Prosody. In: Preminger, Alex; Brogan, Terry V. F. (eds.). The New Princenton Encyclopedia of Poetry and Poetics. Princenton: Princenton University Press, p. 211-215, 1993.

BROWN, Keith; MILLER, Jim. The Cambridge Dictionary of Linguistics. Cambridge: Cambridge University Press, 2013. DOI: https://doi.org/10.1017/cbo9781139049412

CHAICHUMPORN, Jaruchai. Características de la poesía tailandesa. Manuscrito -Universidad de Guadalajara, Guadalajara, 2013.

COSERIU, Eugenio. El problema de la corrección idiomática. Manuscrito inédito resguardado en el Archivo Coseriu de la Universidad de Tübingen - Montevideo, 1956-1957.

COSERIU, Eugenio. Lecciones de lingüística general. Madrid: Gredos, 1981.

COSERIU, Eugenio. Sentido y tareas de la dialectología (Cuadernos de lingüística 8). México, D. F.: Universidad Nacional Autónoma de México, 1982.

COSERIU, Eugenio. Competencia lingüística: Elementos de la teoría del hablar. Madrid: Gredos, 1992 [1988].

FRANK-JOB, Barbara. Diskurstraditionen im Verschriftlichungsprozeß der romanischen Sprachen. In: Aschenberg, Heidi; Wilhelm, Raymund (eds.). Romanische Sprachgeschichte und Diskurstraditionen. Tübingen: Gunter Narr, p. 19-35, 2003.

GALLEGOS SHIBYA, Alfonso. Un acercamiento operacional a la historia de la lengua. Lexis, Lima, v. XXXIV, n. 2, p. 307-350, 2010.

HUDAK, Thomas. Toward an understanding of a Thai poem. In: BICKNER, Robert; HUDAK, Thomas; PEYASANTIWONG, Patcharin. Papers from a Conference on Thai Studies in Honor of William J. Gedney. Ann Arbor: Center for South and Southeast Asian Studies, University of Michigan, p. 185-198, 1986.

ITURRIOZ, José Luis; GÓMEZ, Paula; RAMÍREZ, Xitákame (Julio). Morfología y sintaxis del nombre. In: ITURRIOZ, José Luis (ed.). Lenguas y literaturas indígenas de Jalisco. Guadalajara: Secretaría de Cultura del Gobierno del Estado de Jalisco, p. 143-156, 2004a.

ITURRIOZ, José Luis; GÓMEZ, Paula; RAMÍREZ, Xitákame (Julio). La morfología verbal. In: ITURRIOZ, José Luis. Lenguas y literaturas indígenas de Jalisco. Guadalajara: Secretaría de Cultura del Gobierno del Estado de Jalisco, p. 171-203, 2004b.

ITURRIOZ, José Luis; RAMÍREZ, Julio. El proceso de traducción entre español y huichol. Estudios de traducción, Madrid, v. 2, p. 133-145, 2012. DOI: https://doi.org/10.5209/rev_ESTR.2012.v2.38982

KABATEK, Johannes. Tradiciones discursivas y cambio lingüístico. Lexis, Lima, v. XXIX, n. 2, p. 151-177, 2005.

KABATEK, Johannes. Las tradiciones discursivas entre conservación e innovación. Rivista di filologia e letterature ispaniche, Pisa, v. 10, p. 331-345, 2007.

KABATEK, Johannes. Algunos apuntes acerca de la cuestión de la 'hibridez' y de la 'dignidad' de las lenguas iberorrománicas. In: BLEORȚU, Cristina; GERARDS, David Paul. Lingüística coseriana, lingüística histórica, tradiciones discursivas. Madrid; Frankfurt: Iberoamericana-Vervuert, p. 107-121, 2018a. DOI: https://doi.org/10.31819/9783954877508-007 


\section{REVISTA DA ABRALIN}

KABATEK, Johannes. "Las tradiciones discursivas del español medieval: Historia de textos e historia de la lengua". In: BLEORȚU, Cristina; GERARDS, David Paul (eds.). Lingüística coseriana, lingüística histórica, tradiciones discursivas. Madrid; Frankfurt: Iberoamericana-Vervuert, p. 185-198, 2018b. DOI:

https://doi.org/10.31819/9783954877508-011

KALVERKÄMPER, Hartwig. Rahmenbedingungen für die Fachkommunikation. In: HOFFMANN, Lothar et al. (eds.). Fachsprachen: Languages for Special Purposes, vol. 1. Berlin: de Gruyter, p. 24-47, 1998.

KOCH, Peter. Diskurstraditionen: Zu ihrem sprachtheoretischen Status und ihrer Dynamik. In: FRANK, Barbara et al. (eds.). Gattungen mittelalterlicher Schriftlichkeit. Tübingen: Gunter Narr, p. 43-79, 1997.

KOCH, Peter. Tradiciones discursivas y cambio lingüístico: El ejemplo del tratamiento vuestra merced en español. In: KABATEK, Johannes (ed.). Sintaxis histórica del español y cambio lingüístico: Nuevas perspectivas desde las Tradiciones Discursivas. Madrid; Frankfurt: Iberoamericana-Vervuert, 2008, p. 53-88. DOI: https://doi.org/10.31819/9783865278623-003

LEHMANN, Christian. Nominalisierung: Typisierung von Propositionen. In: SEILER, Hansjakob; LEHMANN, Christian Lehmann (eds.). Apprehension. Das sprachliche Erfassen von Gegenständen. Teil I: Bereich und Ordnung der Phänomene. Tübingen: Gunter Narr, p. 66-83, 1982.

LÓPEZ SERENA, Araceli. Lo universal y lo histórico en el saber expresivo: Variación situacional vs. variación discursiva. In: MARTÍNEZ DEL CASTILLO, Jesús (ed.). Eugenio Coseriu (1921-2002) en los comienzos del siglo XXI. Málaga: Universidad de Málaga, p. 261-281, 2012.

MADDIESON, Ian. Tone. In: HASPELMATH, Martin et al. (eds.). The World Atlas of Language Structures. Oxford: Oxford University Press, p. 58-61, 2005.

OESTERREICHER, Wulf. Zur Fundierung von Diskurstraditionen. In: FRANK, Barbara et al. (eds.). Gattungen mittelalterlicher Schriftlichkeit. Tübingen: Gunter Narr, p. 19-42, 1997.

RAIBLE, Wolfgang. Nominale Spezifikatoren ('Artikel') in der Tradition lateinischer Juristen oder Vom Nutzen einer ganzheitlichen Textbetrachtung für die Sprachgeschichte. Romanistisches Jahrbuch, Berlín, v. 36, p. 44-67, 1985.

SCHIFKO, Peter. ¿Existen lenguas de especialidad? In: BARGALLÓ, María et al. (eds.). Las lenguas de especialidad y su didáctica: Actas del Simposio Hispano-Austriaco. Tarragona: Universitat Rovira i Virgili, p. 21-29, 2001.

SCHLIEBEN-LANGE, Brigitte. Traditionen des Sprechens: Elemente einer pragmatischen Sprachgeschichtsschreibung. Stuttgart: Kohlhammer, 1983.

SCHMITT, Christian. Spanisch: Fachsprachen. In: HOLTUS, Günter et al. (eds.). Lexikon der Romanistischen Linguistik, vol. VI, 1. Tübingen: Niemeyer, p. 295-327, 1992. DOI: https://doi.org/10.1515/9783110939644.295 TUMTAVITIKUL, Apiluck. Thai poetry: A metrical analysis. In: TINGSABADH, M. R. Kanlayā; ABRAMSON, Arthur S. (eds.). Essays in Tai Linguistics. Bangkok: Chulalongkorn University Press, p. 29-40, 2001.

WILHELM, Raymund. Diskurstraditionen. In: HASPELMATH, Martin et al. (eds.) Language Typology and Language Universals: An International Handbook. Berlin: de Gruyter, p. 467-477, 2001.

WILHELM, Raymund. Von der Geschichte der Sprachen zur Geschichte der Diskurstraditionen. Für eine linguistisch fundierte Kommunikationsgeschichte. In: Aschenberg, Heidi; Wilhelm, Raymund (eds.). Romanische Sprachgeschichte und Diskurstraditionen. Tübingen: Gunter Narr, p. 221-236, 2003. 